\title{
Defects in silicon nanowires
}

\author{
R. P. Wang ${ }^{\text {a) }}$ \\ Laser Physics Centre, Research School of Physical Sciences and Engineering, \\ Australian National University, Canberra ACT0200, Australia
}

(Received 24 October 2005; accepted 21 February 2006; published online 4 April 2006)

\begin{abstract}
Defects in silicon nanowires have been investigated using the electron spin resonance (ESR) method. The ESR signals consist of three features: a strong resonance at $g=2.00249$, a weak line at $g=2.00048$, and a broad feature at $g=2.00541$. From the saturation behavior and oxidation-related and temperature dependence analysis, we ascribe that the strong resonance corresponds to the $E X$ center and the weak line to the well-assigned $E^{\prime}$ center. We argue that the assignment of the broad feature to $P_{b}$ centers [A. Baumer et al., Appl. Phys. Lett. 85, 943 (2004)] is oversimplified, and its physical origins may include dangling bonds in amorphous silicon. (C) 2006 American Institute of Physics. [DOI: 10.1063/1.2191830]
\end{abstract}

Over the past decades, defects in silicon-based materials have been intensely investigated because they significantly affect the optical and electrical properties of the materials. A powerful tool to probe the defects is the electron-spinresonance (ESR) spectroscopy. In thermally oxidized silicon, porous silicon, and nanocrystalline silicon, some defects, such as dangling bonds, $E^{\prime}$ center (oxygen vacancy), oxygen shallow donor, and $E X$ center, have been reported, and the correlation between these kinds of defects and various optical and electrical properties has also been elucidated. ${ }^{1-8}$ The renewed interests of ESR study of silicon-based materials are evoked by recent progress in synthesizing freestanding silicon nanowires (SiNWs), which are thought to be the important components in the future nanoscale devices. ${ }^{9,10}$ The investigation of growth mechanisms of SiNWs using transmission electron microscopy (TEM) indicates that a high density of structural defects in silicon nanowires plays an important role in the formation of silicon nanowires and in the determination of the morphology of the nanowires. ${ }^{11}$ The present study pays close attention to the electronically active defects which can be probed by ESR. Although many investigations have been done in silicon-based materials including porous and nanocrystalline silicon structures, the full picture of the physical origins of these defects is still a matter of discussion. By observing the microwave saturation behavior and analyzing oxidation-related and temperature dependences of ESR spectra, we hope to extend the defect studies to identify any defects present and their physical origins in SiNWs.

The SiNW sample was synthesized by an oven-laser ablation method. The bulk quality of SiNWs is perfectly reproducible. The average diameter of the SiNWs used in this study is about $15 \mathrm{~nm}$ and the length varies from a few tens to hundreds of micrometers. The wires consist of a very uniform diameter with a crystalline core of $\sim 10 \mathrm{~nm}$ surrounded by an amorphous coating of $\sim 5 \mathrm{~nm}$. Full details of the experimental results were summarized in previous papers. ${ }^{10-12}$ The ESR experiments were performed at a JEOL system model FA 100 operating at around $9.4 \mathrm{GHz}$. A liquid He flow cryostat was installed in the JEOL system for temperature control. Microwave modulation frequency was kept at

\footnotetext{
${ }^{\text {a)} E l e c t r o n i c ~ m a i l: ~ r p w 111 @ r s p h y s s e . a n u . e d u . a u ~}$
}

$100 \mathrm{KHz}$. Resonance was detected by phase sensitivity detection. As a consequence, the detected signal was the first derivative of absorption curve with respect to the magnetic field.

In total, we have measured several different batches of samples and observed similar ESR signals. The typical ESR spectra at different temperatures are shown in Fig. 1, which indicates that the spectral shape is basically insensitive to the temperature. At room temperature, ESR spectrum consists of three features: First there is a strong resonance at $g=2.00249$ with a linewidth of $0.95 \mathrm{G}$ and there is hyperfine splitting at $323.3 \mathrm{mT}$. Secondly, sometimes we can observe a weak line at $g=2.00048$ which could be ascribed to wellassigned $E^{\prime}$ center in the oxide caused by oxygen vacancies. $2,3,7,13$ Finally, there is a broad ESR feature at $g=2.00541$ with a linewidth of $8.6 \mathrm{G}$.

Regardless of their physical origins, the absolute maximum amplitudes of features A and B in Fig. 1 are used to evaluate the saturation behavior, because ESR measurement below saturation, where the amplitude should be proportional to the microwave magnetic field, is important in order to get the exact information. ${ }^{14}$ We measured the ESR spectra of SiNWs using different microwave powers at room temperature and $77 \mathrm{~K}$. In general, the ESR amplitude increases and then decreases with increasing microwave power as shown in Fig. 2, which displays a linear dependence of amplitude on the square root of the power with increasing mi-

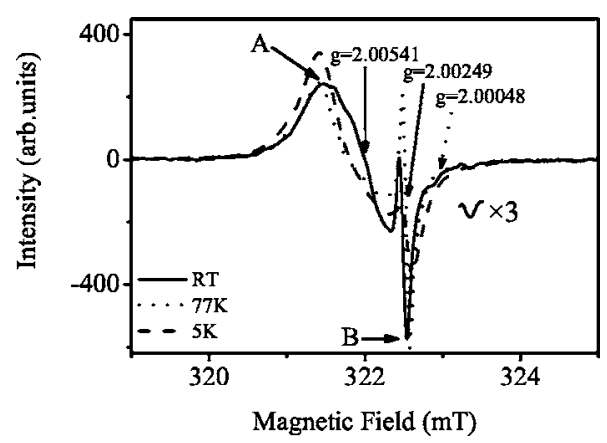

FIG. 1. Typical ESR spectra of SiNWs at different temperatures. The signal intensity was reduced by ten times for $5 \mathrm{~K}$ spectrum and three times for $77 \mathrm{~K}$ spectrum, respectively. The hyperfine splitting line at $323.3 \mathrm{mT}$ was enlarged three times for clarification. All spectra were excited at a microwave power of $0.01 \mathrm{~mW}$. 


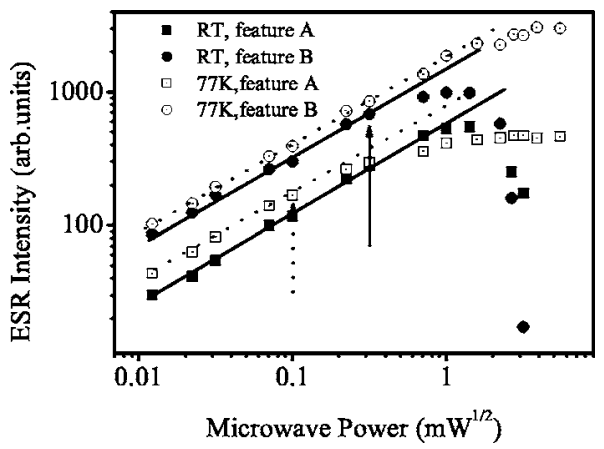

FIG. 2. Saturation curves of ESR signal intensity for applied microwave power.

crowave power below $0.1 \mathrm{~mW}$ at room temperature and $0.01 \mathrm{~mW}$ at $77 \mathrm{~K}$, respectively. The different saturation threshold reveals that the saturation behavior is temperature dependent; therefore in the results reported later, special care has been taken to the measurements with the microwave power below $0.01 \mathrm{~mW}$.

Next, in relation to the origins of these resonance features in Fig. 1, we will focus on two other features instead of the very weak $g=2.00048$ signal that is widely accepted as $E^{\prime}$ center in oxidized or porous silicon. The two features are the sharp resonance line at $g=2.00249$ and another broad feature at $g=2.00541$. Clearly, they show a different behavior at the oversaturation region in Fig. 2, indicating that they come from the different defects.

Concerning the strong resonance line at $g=2.00249$, Stesmans has reported a similar line in thermally oxidized silicon(111). ${ }^{15}$ Carios and Prokes have reported the observation of a sharp isotropic resonance with a $g$ value of 2.00345 in porous silicon, the weak $\mathrm{Si}^{29}$ hyperfine satellites on either side being separated by $16.5 \pm 0.3 \mathrm{G}$ and comprising about $10 \%-12 \%$ of the total integrated intensity. ${ }^{2}$ Baumer et al. found this line at $g=2.00252$ with a hyperfine splitting of $16.1 \mathrm{G}$ in SiNWs grown by thermal vaporization. ${ }^{13}$ The $g$ value and the hyperfine splitting in the room temperature curve of Fig. 1 are quite close to these previous results; therefore we ascribe this strong resonance line to the $E X$ center, which consists of a hole delocalized over three or four oxygen dangling bonds at a silicon vacancy. However, the $E X$ signals found at room temperature are in sharp contrast with those only found at low temperatures in oxidized silicon or porous silicon, ${ }^{2,15}$ suggesting that the number of defects could be dramatically enhanced in the nanomaterials. This is consistent with the existence of a high density of structural defects by TEM observations. ${ }^{11}$

From Fig. 1, we also found a slight broadening of the $E X$ center with decreasing temperature from $0.95 \mathrm{G}$ at room temperature to $1.03 \mathrm{G}$ at $77 \mathrm{~K}$ and $1.25 \mathrm{G}$ at $5 \mathrm{~K}$. When this $E X$ center involves a delocalized spin, previous investigations indicated that the anisotropy of the resonance line may be smeared out by the delocalization because of two different reasons: the extension of the wave function of the spin over several atomic orbitals or a dynamic Jahn-Teller distortion. $^{2,16}$ The linewidth changes with temperature can be expected for the latter. ${ }^{2}$ Carios and Prokes have ascribed it to an extended orbital model although they also mention that there is insufficient experimental evidence. ${ }^{2}$ The present results concerning the change of linewidths seem to support the model of dynamical Jahn-Teller distortion. Downloaded 22 Feb 2007 to 150.203.178.183. Redistribution subject to AlP license or copyright, see http://apl.aip.org/apl/copyright.jsp

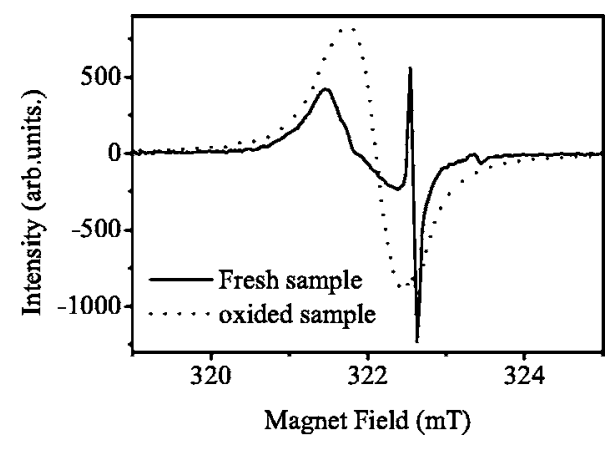

FIG. 3. ESR spectra of fresh and oxidized SiNWs at room temperature.

For the broad resonance at $g=2.00541$, Baumer et al. ascribed it to $P_{b}$ centers located at the interface of the crystalline core and the surrounding oxide; ${ }^{13}$ Prokes et al. measured ESR spectra of nanocrystal $\mathrm{Si} / \mathrm{SiO}_{2}$ structure processed by various oxidation conditions and concluded that the broad ESR feature is due to an oxygen-related center whose formation may be enhanced during long-time annealing stages. ${ }^{3}$ To understand this broad ESR feature, we measured ESR spectra of fresh and oxidized samples and the results are shown in Fig. 3. After the SiNW sample was exposed to atmosphere at room temperature for several months, we found that Raman scattering peak around $520 \mathrm{~cm}^{-1}$, which is a symbol of crystalline silicon structure, is greatly suppressed, indicating that most of the material become amorphous. ${ }^{12}$ This amorphous material is defective because the oxidization process happens at very low temperatures. For such sample, the broad feature in normal SiNW ESR spectrum is enhanced while the strong EX resonance almost disappears as shown in Fig. 3. This evolutionary trend of ESR signals is quite similar to that of porous silicon that is oxidized in dry $\mathrm{O}_{2}$ at $800{ }^{\circ} \mathrm{C}$ for $1 \mathrm{~min}$ and also to that of oxidized $\mathrm{Si} / \mathrm{SiO}_{2}$ nanostructure, ${ }^{2,3}$ but is in sharp contrast to that of HF-treated SiNWs where all ESR features decrease drastically. ${ }^{14}$ ESR study of oxidized and H-terminated SiNWs reveals that the ESR signal is highly oxygen related, and therefore previous assignment of this broad peak to possible $P_{b}$ centers located at the interface of the crystalline core and the surrounding oxide seems oversimplified. The strongest position in this broad feature in Fig. 3 shifts to a low $g$ value and becomes broad after oxidization, suggesting that this feature is comprised of more than one resonance.

The temperature dependence of the shift of the broad feature in Fig. 1 provides another evidence that this feature is comprised of two (or more) defect centers. We argue that the $P_{b}$ centers and the dangling bonds in amorphous silicon are dominant in this broad feature although some unknown defects may contribute to it. Umeda et al. have investigated this kind of defects in undoped $a-\mathrm{Si}: \mathrm{H}$ and concluded that the dangling bond center is localized predominantly on a single silicon atom and is characterized as strongly $p$ like. It has quite a similar bonding structure, electronic structure, and site-to-site variation to the dangling bonds at the $\mathrm{Si}(111) / \mathrm{SiO}_{2}$ interface except for a much weaker localization. ${ }^{17}$ We believe that the SiNW structure may include a number of amorphous silicon between crystalline core and the surrounding $\mathrm{SiO}_{2}$ layer. We note that even high resolution TEM image cannot indicate the difference between amorphous silicon and $\mathrm{SiO}_{2}$, and also there is no de- 
tailed report about the evolution of the chemical composite along the radial direction of the SiNWs. An investigation using electron energy loss spectrum at small regions of around several nanometers may resolve this issue. However, since all SiNWs were prepared in vacuum, the oxygen deficiency could be expected for all wires and oxygen distribution should gradually decrease to zero at the interface along the radial direction, leading to amorphous silicon registering at this regions. When ESR measurements are performed at low temperatures, the weak localization of dangling bonds in amorphous silicon is enhanced, therefore the broad resonance line shifts to high $g$ values. On the other hand, when the SiNW sample is oxidized at room temperature, amorphous silicon and the crystalline core are suppressed and the ratio of surface to volume increases. Therefore, the number of $P_{b}$ centers at the interface of the crystalline core and the surrounding oxide increases, and ESR signals are enhanced and shift to low $g$ values as shown in Fig. 3 .

In summary, we have investigated the defects present in SiNWs using ESR method. We ascribe the weak resonance line at $g=2.00048$ to the well-assigned $E^{\prime}$ center. By studying the saturation behavior and oxidation-related and temperature dependences of ESR spectra, we conclude that the strong resonance line at $g=2.00249$ corresponds to the $E X$ center, and previous assignment of the broad feature to $P_{b}$ centers is oversimplified. We argue that the possible physical origin of the broad feature may include dangling bonds in amorphous silicon.

${ }^{1}$ M. Cook and C. T. White, Phys. Rev. Lett. 59, 1741 (1987).

${ }^{2}$ W. E. Carios and S. M. Prokes, J. Appl. Phys. 78, 2129 (1995).

${ }^{3}$ S. M. Prokes, W. E. Carlos, S. Veprek, and Ch. Ossadnik, Phys. Rev. B 58, 15632 (1998).

${ }^{4}$ N. Ishii and T. Shimizu, Phys. Rev. B 42, 9697 (1990).

${ }^{5}$ T. Umeda, S. Yamasaki, J. Isoya, and K. Tanaka, Phys. Rev. B 62, 15702 (2002).

${ }^{6}$ M. Fujii, A. Mimura, S. Hayashi, Y. Yamamoto, and K. Murakami, Phys. Rev. Lett. 89, 206805 (2002).

${ }^{7}$ M. Dohi, H. Yamatani, and T. Fujita, J. Appl. Phys. 91, 815 (2002).

${ }^{8}$ M. M. De Lima, Jr., P. C. Taylor, S. Morrison, A. LeGeune, and F. C. Marques, Phys. Rev. B 65, 235324 (2002).

${ }^{9}$ A. M. Morales and C. M. Lieber, Science 279, 208 (1998).

${ }^{10}$ D. P. Yu, C. S. Lee, I. Bello, G. W. Zhou, and Z. G. Bai, Solid State Commun. 105, 403 (1998).

${ }^{11}$ G. Zhou, Z. Zhang, Z. Bai, S. Feng and D. Yu, Appl. Phys. Lett. 73, 677 (1998).

${ }^{12}$ R. P. Wang, G. W. Zhou, Y. L. Liu, S. H. Pan, and Z. Zhang, Phys. Rev. B 61, 16827 (2000).

${ }^{13}$ A. Baumer, M. Stutzmann, M. S. Brandt, F. C. K. Au, and S. T. Lee, Appl. Phys. Lett. 85, 943 (2004).

${ }^{14}$ C. P. Poole, Jr., Electron Spin Resonance (Dover, New York, 1996).

${ }^{15}$ A. Stesmans, Phys. Rev. B 45, 9501 (1992).

${ }^{16}$ A. Stesmans and F. Scheerkinck, Phys. Rev. B 50, 5204 (1994).

${ }^{17}$ T. Umeda, S. Yamasaki, J. Isoya, and K. Tanaka, Phys. Rev. B 59, 4849 (1999). 